\title{
Epigenetic silencing of PTEN and epi-transcriptional silencing of MDM2 underlied resistance to hypomethylating agents in myelodysplastic syndrome
}

\section{Paul Lee}

the University of Hong Kong

Rita Yim

the University of Hong Kong

Kai-Kei Miu

the Chinese University of Hong Kong

Sin-Hang Fung

the Chinese University of Hong Kong

Jason Jinyue Liao

the Chinese University of Hong Kong

Zhangting Wang

the Chinese University of Hong Kong

Jun Li

the City University of Hong Kong

Yammy Yung

the University of Hong Kong

Hiu-Tung Chu

the University of Hong Kong

\section{Pui-Kwan Yip}

the University of Hong Kong

Emily Lee

the University of Hong Kong

Eric Tse

the University of Hong Kong

Yok-Lam Kwong

the University of Hong Kong

Harinder Gill ( $\nabla$ gillhsh@hku.hk )

the University of Hong Kong 


\section{Research Article}

Keywords:

Posted Date: April 19th, 2022

DOI: https://doi.org/10.21203/rs.3.rs-1274380/v2

License: (c) (i) This work is licensed under a Creative Commons Attribution 4.0 International License. Read Full License 


\section{Abstract}

\section{Background}

In myelodysplastic syndrome (MDS), resistance to hypomethylating agents (HMA) portends a poor prognosis, underscoring the importance of understanding the molecular mechanisms leading to HMAresistance.

\section{Methods}

In this study, P39 and Kasumi-1 cells and their azacitidine-resistant and decitabine-resistant sublines were evaluated comparatively with transcriptomic and methylomic analyses.

\section{Results}

Expression profiling and genome-wide methylation microarray showed downregulation of PTEN associated with DNA hypermethylation in P39 cell lines resistant to azacitidine and decitabine. This pattern of PTEN dysregulation was also confirmed in a cohort of patients failing treatment with HMA. DNA hypomethylation of MDM2 was detected with downregulation of MDM2 in HMA resistant cell lines. Long-read sequencing revealed significant RNA hypomethylation of $M D M 2$ resulting in alternative splicing and production of a truncated MDM2 transcript in azacitidine-resistant P39 cells. The expression of this MDM2 truncated transcript was also significantly increased in HMA-resistant patients compared with HMA-responsive patients.

\section{Conclusions}

In conclusion, epigenetic and epi-transcriptomic dysregulation of PTEN and MDM2 were associated with resistance to hypomethylating agents.

\section{Introduction}

Myelodysplastic syndrome (MDS) is a clonal myeloid neoplasm characterized by multiple genetic aberrations and complex clonal architectures[1]. These diverse genetic aberrations account for the different clinical presentations, including treatment responses, survivals and risks of progression to acute myeloid leukemia (AML)[2]. The hypomethylating agents (HMA) azacitidine (AZA) and decitabine (DEC) are standard therapeutic options in MDS. Therapeutic objectives in MDS include ameliorating cytopenia and related complications, reducing transfusion requirement, enhancing quality of life, lowering risks of secondary AML and improving survivals[3,4].

Responses to AZA and DEC in patients with MDS and AML with myelodysplasia-related changes, are generally short-lived[5]. Somatic mutations of ASXL1, TET2 and/or DNMT3A are associated with a hypermethylated genomic profile, which alters responses to HMA[5-8]. Studies at the messenger RNA (mRNA) level showed that resistance to AZA was associated with downregulation of uridine-cytidine 
kinase (UCK), an enzyme required for AZA activation upon cellular uptake[9]. In studies evaluating resistance to $D E C$, a higher expression ratio of cytidine deaminase $(C D A)$ to deoxycytidine kinase $(D C K)$ impaired mono-phosphorylation and hence activation of DEC, with accelerated deamination of DEC leading to faster rate of DEC inactivation[10]. Targeted methylation analyses also showed hypomethylation of LINE1, PGRB and miR-124a-3, but the pathogenetic roles of these genes remain unclear[10].

Although gene dysregulations associated with HMA resistance have been widely studied, a comprehensive approach that correlates somatic mutations and epigenetic alterations with gene activities may reveal important mechanisms accounting for HMA resistance[5, 11, 12]. In this study we employed a multi-omics approach to identify biologic pathways altered during HMA resistance in cell lines and patient samples. In addition to genome-wide methylation analysis, the transcriptome and epitranscriptome were evaluated for expression, somatic mutations and RNA methylation, aiming to correlate transcriptomic dysregulation with genetic, epigenetic, and epi-transcriptional signatures.

\section{Methods}

\section{Cell lines and cell cultures}

P39/Tsugane (courtesy of Prof Yoshida Takeda, Japan) and Kasumi-1 (DSMZ) cell lines were cultured using medium RPMI-1640 glutamax-1 with 10\% fetal bovine serum (FBS) and RPMI-1640 with 20\% FBS respectively. Penicillin $(25 \mathrm{IU} / \mathrm{mL})$ and streptomycin $(25 \mu \mathrm{g} / \mathrm{mL})$ were supplemented and cells were incubated at $37^{\circ} \mathrm{C}$ in humidified $5 \% \mathrm{CO}_{2}$. To establish derivative sublines resistant to AZA and DEC, both cell lines were continuously exposed to increasing concentrations of AZA and DEC from 5nM onwards. Sublines that were resistant to $\geq 10 \mu \mathrm{M}$ of AZA or DEC was established as P39-AZA-R and P39-DEC-R, and Kasumi-1-AZA-R and Kasumi-1-DEC-R.

\section{Cell viability assays}

To determine the IC50 of AZA and DEC for the parental cell lines and sublines, cells were seeded at a density of $1000 / \mu \mathrm{L}$ and treated with increasing doses of AZA and DEC. Cell viability was determined with presto blue stain at 24,48 and 72 hours during treatment with $2.5 \mathrm{nM}$ to $2.5 \mathrm{mM}$ of $A Z A$ or $1 \mathrm{nM}$ to 0.1 mM DEC. The IC50s of AZA and DEC were determined.

\section{DNA whole methylome analysis}

The Illumina ® Infinium MethylationEPIC BeadChip (850k) (Illumina, San Diego, USA) was used to quantify $866,836 \mathrm{CpG}$ sites in the genome according to the manufacturer's protocol. Briefly, bisulfite conversion of genomic DNA was performed using Zymo EZ DNA methylation kit (Zymo Research, Irvine, USA) and denatured for isothermal whole genome amplification. Amplified DNA was fragmented, and hybridized overnight onto BeadChip followed by washing, staining and extension. The final chip imaging was performed on Illumina ${ }^{\circledR}$ iScan System (Illumina, San Diego, USA). Bioinformatic analysis of

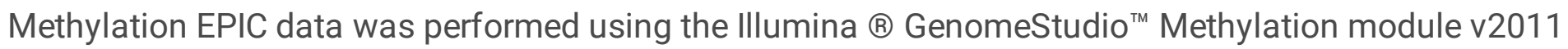


(Illumina, San Diego, USA). CpG sites passing quality controls were filtered and normalized for differential methylation calculation. Comparison between P39 (control group) and P39-AZA-R and P39-DEC-R (resistant group) lines were made with threshold above 2.5-fold or below 0.4-fold. Unsupervised clustering, Gene ontology (GO) and Kyoto Encyclopedia of Genes and Genomes (KEGG) analyses using the selected gene list with differential methylation regions (DMRs) were performed and visualized[13, 14].

\section{Whole transcriptome analysis}

High quality RNA extracted from P39, P39-AZA-R, P39-DEC-R lines was enriched for mRNA by Oligo (dT) followed by preparation into whole transcriptomic library. Paired-end sequencing was performed on the Beijing Genome Institute (BGI, ShenZhen, China) next-generation sequencing platform (BGISEQ-500) to acquire at least 5 million reads at 100bp per read. Cleaned reads pre-processed by SOAPnuke toolkit were mapped to the reference genome hg19, while spliced-aligned reads mapped by HISAT2 were subjected to StringTie and Cuffcompare to reconstruct transcripts for novel transcript prediction[15-18]. The combined transcriptomic assembly was used as reference for reads re-alignment by Bowtie2[19] followed by gene expression level calculation with RNA-seq by Expectation Maximization (RSEM)[20].

Subsequent hierarchical clustering and Pearson correlation were analysed using default packages cor and hclust in $\mathrm{R}$ and heatmaps were visualized using ggplot in R[21]. Differentially expression genes (DEG) with more than 2-fold difference between P39 and P39-AZA-R / P39-DEC-R lines were identified using the PossionDis software. Functional annotations and predictions were conducted using Gene Ontology (GO) analysis and KEGG pathway analysis to detect enriched functional GO terms, biological processes and signalling pathways. Distribution analysis was performed using phyper to monitor threshold false discover rates (FDR), with significant enriched GO terms defined as no larger than 0.01 $[14,22,23]$. SNPs and INDELs were detected following the GATK method and gene fusion events were detected using SOAPfuse[24]. High confidence variants were filtered by default parameter and the Circos software was used to visualize gene fusions[25].

\section{RNA modifications and alternative splicing detection}

Native RNA libraries were prepared from P39 and P39-AZA-R lines with the direct RNA sequencing kit (Oxford Nanopore Technologies, Oxford, United Kingdom) according to the manufacturer's protocol (SQKRNA002). Briefly, reverse-transcribed RNA was purified and loaded into flow cell (FLO-MIN106D) for sequencing. Raw electrical signal data (.fast5) were used for base calling using guppy (v4.0.5, Oxford Nanopore Technologies) with the high accuracy model. The quality-filtered direct RNA-seq (DRS) reads were mapped to GrCh38 reference human transcriptome with minimap2 (v2.17) utilizing default parameters that detected spliced long reads[26]. The resulting data were resquiggled with nanopolish eventalign (v0.13.2)[27]. For the detection of differential RNA modifications, xpore (v0.5.3) was adopted with default parameters[28].

\section{Pyrosequencing and quantitative polymerase chain reaction}


Hypermethylated or hypomethylated loci of interest were validated by pyrosequencing (Supplemental File 1). Completely methylated and completely unmethylated controls (Qiagen, Maryland, United States) were used and mixed in 1:1 ratio to produce a $50 \%$ methylated control. Gene expression quantification by Real Time-PCR was performed using the SuperScript IV VILO Master Mix (Thermo Fisher, Massachusetts, United States) and PowerUp ${ }^{\mathrm{TM}}$ SYBR ${ }^{\mathrm{TM}}$ Green Master Mix (Thermo Fisher Scientific, Massachusetts, United States).

\section{Patients and samples}

Bone marrow (BM) aspirates of 13 patients with MDS resistant to HMA and 14 MDS patients responsive to HMA were used Resistance to HMA was defined as disease progression from MDS to secondary AML during HMA treatment, and BM samples at progression were collected. Responsiveness to HMA was defined as marrow and / or haematological improvement. BM samples at the time of progression to AML and at best response to HMA were collected. Baseline clinicopathologic and molecular characteristics of all patients were determined and reviewed before being used to validate cell line data generated in vitro. This study was approved by the Institutional Review Board of the University of Hong Kong/ Hong Kong West Cluster (UW 19-634) and written informed consents were obtained.

\section{Statistical analyses and data sharing}

Unless otherwise specified, all grouped data were presented as mean \pm standard error (SE). Statistical analyses were performed using the unpaired t-test or Chi-square test. Correlation between variables were determined using the Pearson correlation coefficient. Two-tailed $p$-values of $<0.05$ were considered statistically significant. All high-throughput sequencing data that supported the findings of this study were deposited in the Gene Expression Omnibus (GEO) with the accession code (GSE165188). For specific dataset, the accession numbers are GSE165185 for methylation microarray data, GSE165187 for RNA transcriptomic data, and GSE165064 for native RNA sequencing data for epi-transcriptomic analysis. Other relevant data supporting the key findings of this study are available within the article or upon request to the corresponding author.

\section{Results}

\section{Establishment of AZA-R and DEC-R sublines}

By continuously exposing P39 and Kasumi-1 cells to AZA or DEC, P39-AZA-R and Kasumi-1-AZA-R and P39-DEC-R and Kasumi-1-DEC-R sublines were established. The IC50s of the P39-AZA-R / P39-DEC-R and Kasumi-1-AZA-R / Kasumi-1-DEC-R sublines were $\geq 1$.5-log and $\geq 2$ log-fold higher than those of P39 and Kasumi-1 (supplemental file 2). Cells resistant to AZA and DEC were harvested for whole methylome and transcriptome analysis.

\section{Methylome dysregulation during HMA resistance in cell lines}


Of the 866,836 CpG sites tested, 3604 loci were hypermethylated in P39-AZA-R and/or P39-DEC-R cells, with an average methylation levels of $\geq 2.5$-fold higher than those of P39 cells. In contrast, there was at least 10-fold more hypomethylated loci (45,932 CpG sites) with average methylation level $\leq 0.4$-fold in HMA-resistant cells compared with HMA-sensitive parental cells (Fig. 1A). Amongst the 20,126 annotatable differential methylation regions (DMRs), 33\% (i.e. 6689 loci) were classified as CpG islands while the remaining were CpG-flanking sites of either CpG shelf or shore (supplemental files 3 and 4). When studying the DMRs in the context of localization to known UCSC RefGene, the majority of CpGs were embedded within gene bodies while there were $32 \%$ either in close vicinity to the transcriptional start site (TSS) or spanning the first exon (supplemental files 3 and 4).

Genome-wide hypomethylation in both P39-AZA-R and P39-DEC-R was also observed in the clustering analysis with minor differences between P39-AZA-R and P39-DEC-R (Fig. 1B). In particular, among the 45,932 hypomethylated $\mathrm{CpGs}$, there were 42\% (19,498 hypomethylated $\mathrm{CpGs}$ ) predicted either in close vicinity to TSS or spanning the first exon of the known genes or non-coding RNAs. Excluding the 555 CpGs mapping to unclassifiable open reading frames, there were 18941 hypomethylated CpGs mapping to known gene or non-coding RNA (supplemental file 5). Despite global hypomethylation signatures in both P39-AZA-R and P39-DEC-R, 88\% of the hypermethylated CpGs (3170 loci) were predicted to be either in close proximity to TSS or spanning the first exon of UCSC RefGene known genes or non-coding RNA.

Among the 11,564 genes or non-coding RNA with differential methylation signatures, PTPRN2, AKNRD11, MAD1L1, RPTOR, TNXB were the genes with the highest frequencies of differential methylation while PTEN and MDM2 also showed significant differential methylation (supplemental file 6). Results from the GO and KEGG prediction analyses indicated that TP53 dysregulation was implicated. TP53 function was significantly associated with the PI3K/Akt/mTOR signalling pathway, with PTEN and MDM2 predicted to play major roles (supplemental file 7). In addition to PTEN and MDM2, other genes predicted to be associated with HMA resistance by GO and KEGG analyses included EGFR, p16INK4a (CDKN2A) and p14ARF, all of which were directly or indirectly regulated by PTEN and MDM2.

\section{Transcriptomic dysregulation and corresponding DNA methylomic changes in HMA-resistant cell lines}

P39-AZA-R and P39-DEC-R displayed distinct transcriptomic profiles in the two clustering analyses (Figs. 2A and 2B). There were significantly more upregulated genes in P39-DEC-R when compared with P39-AZA-R (1296 versus 306 upregulated genes), while there were only 89 downregulated genes in P39DEC-R, 331 genes fewer than that observed in P39-AZA-R (supplemental file 8 and supplemental file 9). Among the downregulated genes in P39-AZA-R and/or P39-DEC-R, 10 genes were found to be hypermethylated based on whole methylome analysis, including KIFC1, CTTN, FAM171A2, EPAS1, KANK2, PDGFA, KIFC3, CDKN1C, TP53INP2, and FTH1. During functional prediction of these commonly dysregulated genes, the KEGG pathway analysis predicted pathogenic activation of multiple signaling pathways, most notably the PI3K/Akt/mTOR signaling pathway (Fig. 3). GO terms classification analysis indicated that the majority of identified genes were involved in cellular processing, and approximately 
$50 \%$ of dysregulated genes were involved in signaling transduction via protein-protein binding or proteinnucleic acid binding activities (supplemental file 10).

Aberrant DNA methylation associated with PTEN and MDM2 dysregulation in HMA-resistant cell lines

Amongst all genes, dysregulation of PTEN and MDM2, and the PI3K/Akt/mTOR signaling pathway, was consistently predicted to be significant by both methylomic and transcriptomic analyses. PTEN and MDM2 showed differential methylation and RNA expression in P39 during resistance to HMA and were functionally significant based on predictions made by KEGG and GSEA analyses (Fig. 3 and supplemental file 7). PTEN and MDM2 were therefore selected for validation by pyrosequencing and realtime PCR in parental and HMA-resistant lines. Among the hypermethylated loci, cg03891929 and cg10041390 located inside the two CpG islands were approximately $200 \mathrm{bp}$ upstream to PTEN, and they were hypermethylated by 2.9-fold and 4.4-fold respectively in HMA-resistant lines. For pyrosequencing spanning cg10041390 and the eight flanking CpGs, a low level of methylation was detected in HMA resistant P39 cells. The average methylation across all CpG loci of both P39-AZA-R and P39-DEC-R were higher than that of P39 ( $p=0.0028$ and $p=0.02$ respectively). In particular, P39-AZA-R showed a 10-40\% increase of methylation in comparison with P39 and hypermethylation of P39-DEC-R was observed across all sites analysed except cg10041390, ranging from 3-21\% (Table 1 and supplemental file 11). 
Table 1

PTEN methylation of cell lines sensitive to and resistant to hypomethylating agents.

\begin{tabular}{|c|c|c|c|c|c|c|c|c|c|c|}
\hline Cell line* & $\begin{array}{l}\text { CpG } \\
1 \\
(\%)\end{array}$ & $\begin{array}{l}\text { CpG } \\
2 \\
(\%)\end{array}$ & $\begin{array}{l}\text { CpG } \\
3 \\
(\%)\end{array}$ & $\begin{array}{l}\text { Cpg } \\
4(\%)\end{array}$ & $\begin{array}{l}\text { CpG } \\
5 \\
(\%)\end{array}$ & $\begin{array}{l}\text { CpG } \\
6 \# \\
(\%)\end{array}$ & $\begin{array}{l}\text { CpG } \\
7 \\
(\%)\end{array}$ & $\begin{array}{l}\text { CpG } \\
8 \\
(\%)\end{array}$ & $\begin{array}{l}\text { CpG } \\
9(\%)\end{array}$ & $\begin{array}{l}\text { Mean } \\
(\%)\end{array}$ \\
\hline $\begin{array}{l}\text { Parental P39 } \\
\text { sensitive to } A Z A^{\star *}\end{array}$ & 5.95 & 7.78 & 7.41 & 11.91 & 7.23 & 3.96 & 3.54 & 6.04 & 14.1 & 7.55 \\
\hline $\begin{array}{l}\text { Parental P39 } \\
\text { sensitive to DEC** }\end{array}$ & 4.91 & 6.5 & 6.91 & 12.37 & 7.57 & 4.39 & 3.8 & 6.7 & 13.7 & 7.43 \\
\hline P39-AZA-R & 4.87 & 7.46 & 6.82 & 13.07 & 8.69 & 4.67 & 5.01 & 7.22 & 15.16 & 8.11 \\
\hline P39-DEC-R & 5.47 & 7.88 & 8.07 & 12.93 & 8.4 & 4.35 & 3.94 & 6.87 & 15.16 & 8.12 \\
\hline $\begin{array}{l}\text { Parental Kasumi-1 } \\
\text { sensitive to } A Z A^{\star \star}\end{array}$ & 5.86 & 7.98 & 5.61 & 11.57 & 7.56 & 4.53 & 4.22 & 6.09 & 13.48 & 7.43 \\
\hline $\begin{array}{l}\text { Parental Kasumi-1 } \\
\text { sensitive to DEC** }\end{array}$ & 4.86 & 7.69 & 5.35 & 11.75 & 6.66 & 4.31 & 4.54 & 6.89 & 14.45 & 7.39 \\
\hline Kasumi-1-AZA-R & 6.56 & 8.52 & 7.35 & 15.41 & 7.77 & 5.67 & 6.53 & 8.21 & 18.82 & 9.43 \\
\hline Kasumi-1-DEC-R & 4.61 & 7.26 & 5.74 & 11.18 & 6.07 & 4.19 & 3.74 & 5.52 & 12.34 & 6.74 \\
\hline \multicolumn{11}{|c|}{$\begin{array}{l}\text { * P39, Kasumi-1 and their derivative cell lines resistant to either azacitidine or decitabine were } \\
\text { analyzed for PTEN methylation by pyrosequencing. }\end{array}$} \\
\hline \multicolumn{11}{|c|}{$\begin{array}{l}\text { ** The sensitive parental P39 and Kasumi- } 1 \text { cells were treated separately with AZA and DEC at } 1 \mu \mathrm{M} \\
\text { for } 48 \text { hours followed by immediate harvest for the assessment }\end{array}$} \\
\hline \multicolumn{11}{|c|}{$\begin{array}{l}\text { \#: The sixth CpG site analyzed corresponded to the same loci (cg10041390) detected to be } \\
\text { hypermethylated in } 850 \mathrm{k} \text { whole methylome microarray. }\end{array}$} \\
\hline \multicolumn{11}{|c|}{$\begin{array}{l}\text { P39-AZA-S: Azacitidine-sensitive P39 cell line; P39-DEC-S: Decitabine-sensitive P39 cell line; P39-AZA- } \\
\text { R: Azacitidine-resistant P39 cell line; P39-DEC-R: Decitabine-resistant P39 cell line; Kasumi-1-AZA-S: } \\
\text { Azacitidine-sensitive Kasumi-1 cell line; Kasumi-1-DEC-S: Decitabine-sensitive Kasumi-1 cell line; } \\
\text { Kasumi-AZA-R: Azacitidine-resistant Kasumi-1 cell line; Kasumi-1-DEC-R: Decitabine-resistant Kasumi- } \\
1 \text { cell line. }\end{array}$} \\
\hline
\end{tabular}

In addition to the P39 lines, PTEN hypermethylation pattern was only observed in Kasumi-AZA-R ( $\mathrm{p}=$ 0.001 ) but not Kasumi-1-DEC-R, which showed hypomethylation at most CpG loci $(p=0.003)$ (Table 1 and supplemental file 12).

When all cell lines were studied for PTEN mRNA expression, all four HMA-resistant cell lines showed significant downregulation of PTEN compared with parental cells (Fig. 4A).

For MDM2, three hypomethylated CpG loci cg00614420, cg09781971 and cg24703804 were examined insilico for the presence of $\mathrm{CpG}$ island or shores. Only cg00614420 and one adjacent CpG site mapping approximately $900 \mathrm{bp}$ upstream to MDM2 were validated by pyrosequencing (Table 2 and supplemental file 13). Among all four HMA-resistant cell lines, hypomethylation of both $\mathrm{CpG}$ sites were observed with 
significance (all $\mathrm{p}$ <0.05). When genomic MDM2 hypomethylation was correlated with MDM2 transcriptional activity, MDM2 mRNA expression level dropped in all resistant cell lines in comparison with their corresponding parental cells $(p<0.05$, Fig. 4D).

Table 2

MDM2 methylation of cell lines sensitive to and resistant to hypomethylating agents.

\begin{tabular}{|c|c|c|c|}
\hline Cell line* & CpG 1 (\%) & CpG $2(\%) \#$ & Mean (\%) \\
\hline Parental P39 sensitive to $A Z A^{\star \star *}$ & 45.92 & 28.05 & 36.99 \\
\hline Parental P39 sensitive to DEC** & 56.31 & 32.24 & 44.28 \\
\hline P39-AZA-R & 26.37 & 16.77 & 21.57 \\
\hline P39-DEC-R & 31.44 & 21.71 & 26.58 \\
\hline Parental Kasumi- 1 sensitive to $A Z A^{\star *}$ & 57.97 & 35.89 & 46.93 \\
\hline Parental Kasumi- 1 sensitive to DEC** & 66.45 & 42.91 & 54.68 \\
\hline Kasumi-1-AZA-R & 37.49 & 19.56 & 28.53 \\
\hline Kasumi-1-DEC-R & 44.07 & 26.95 & 35.51 \\
\hline \multicolumn{4}{|c|}{$\begin{array}{l}\text { * P39, Kasumi-1 and their derivative cell lines resistant to either azacitidine or decitabine were } \\
\text { analyzed for MDM2 methylation by pyrosequencing. }\end{array}$} \\
\hline \multicolumn{4}{|c|}{$\begin{array}{l}\star \star \text { The sensitive parental P39 and Kasumi- } 1 \text { cells were treated separately with AZA and DEC at } 1 \mu \mathrm{M} \\
\text { for } 48 \text { hours followed by immediate harvest for the assessment }\end{array}$} \\
\hline \multicolumn{4}{|c|}{$\begin{array}{l}\text { \# The second CpG analyzed by pyrosequencing was the same loci (cg00614420) detected } \\
\text { hypomethylated in } 850 \mathrm{k} \text { whole methylome microarray. }\end{array}$} \\
\hline \multicolumn{4}{|c|}{$\begin{array}{l}\text { P39-AZA-S: Azacitidine-sensitive P39 cell line; P39-DEC-S: Decitabine-sensitive P39 cell line; P39-AZA- } \\
\text { R: Azacitidine-resistant P39 cell line; P39-DEC-R: Decitabine-resistant P39 cell line; Kasumi-1-AZA-S: } \\
\text { Azacitidine-sensitive Kasumi-1 cell line; Kasumi-1-DEC-S: Decitabine-sensitive Kasumi-1 cell line; } \\
\text { Kasumi-AZA-R: Azacitidine-resistant Kasumi-1 cell line; Kasumi-1-DEC-R: Decitabine-resistant Kasumi- } \\
1 \text { cell line. }\end{array}$} \\
\hline
\end{tabular}

To explore alternative reasons for downregulated MDM2 expression, the somatic mutation profile was examined in the RNA sequencing data, with no MDM2 mutation detectable. The SNP distributions across known gene elements (5'-UTR, exon, intron, 3'-UTR and intergenic region) also showed no significant changes in HMA-resistant P39 sublines (supplemental file 14). On the other hand, a significant decrease in the number of fusion events was observed in both P39-AZA-R and P39-DEC-R, with only 2 and 4 fusions event remaining in P39-AZA-R and P39-DEC-R respectively (supplemental file 15). The fusion genes observed in both sensitive and resistant lines included CLN6-CALML4, SRGAP2B-SRGAP2C and CYFIP2-PLCG2.

\section{Epi-transcriptomic profiling of P39 and P39-AZA-R}


To explore potential mechanisms alternative to DNA methylation that might be involved in MDM2 downregulation in HMA-resistant P39 cells, whole epi-transcriptomic profiling was performed. Direct longread sequencing of P39 and P39-AZA-R detected 5,340,619 differential methylated $\left(\mathrm{m}^{6} \mathrm{~A}\right)$ RNA loci and $\mathrm{GO}$ analysis predicted that the majority of dysregulated transcripts were associated with ribonucleoprotein biogenesis and neutrophil function (Fig. 4G). Meanwhile, RNA splicing was the third most enriched pathway and one of the MDM2 processed transcripts (ENST00000400501.2) was found to be significantly hypomethylated by 0.19 -fold in P39 compared with P39-AZA-R $\left(p=1.12 \times 10^{-9}\right)$. This truncated transcript with predicted length of $406 \mathrm{bp}$ was predicted to be non-protein coding, with the RING domain absent in comparison with normal MDM2. The processed MDM2 transcript was quantified in P39 and Kasumi-1 with their respective AZA-R and DEC-R sublines (Fig. 4H). Results showed that the MDM2 processed transcript was upregulated in P39-AZA-R $(p=0.03)$, with a trend of upregulation also observed in P39-DEC-R and Kasumi-1-DEC-R ( $p=0.33$ and 0.18 respectively) in comparison with the parental lines. Interestingly, Kasumi-AZA-R showed a significant downregulation of the MDM2 processed transcript $(\mathrm{p}=$ 0.003).

\section{Validation in primary MDS patient samples}

The two important findings based on the multi-omic analyses in cell lines were validated in BM samples of patients with higher-risk MDS (Supplemental file 16). Firstly, results from the HMA-resistant P39 sublines suggested that DNA methylation mediated silencing of PTEN. In BM samples, all CpG loci in PTEN including cg10041390 showed significantly higher methylation levels in HMA-resistant patients compared with HMA-responsive patients (Fig. 4B, $\mathrm{p}=0.004$ ). Furthermore, PTEN expression was significantly downregulated in HMA-resistent patients compared with HMA-responsive patients (Fig. 4C, $p$ $=0.042$ ). Secondly, in HMA-resistant sublines, hypomethylation of the CpG loci cg00614420 and one adjacent CpG site mapping approximately 900bp upstream in MDM2 was observed. Similarly, pyrosequencing showed significant hypomethylation of the same $\mathrm{CpG}$ sites in BM samples from HMAresistant patients compared with those observed in HMA-responsive patients $(p=0.040)$ (Fig. 4E). In contrast to the observation in HMA-resistant cell lines, MDM2 expression was not significantly downregulated $(p=0.152)$ (Fig. 4F) in HMA-resistant patients compared with HMA-reponsive patients. The expression of the MDM2 processed transcript was also significantly increased in HMA-resistant patients compared with HMA-responsive patients $(p=0.008)($ Fig. 4I).

\section{Discussion}

In this study, transcriptome, methylome and epitranscriptome were studied together to decipher the molecular mechanisms contributing to HMA resistance. In the MDS/secondary AML cell line P39, we showed consistent downregulation of PTEN and MDM2 and increased expression of a truncated MDM2 transcript during HMA resistance. However, in the comparator AML line Kasaumi-1, which contained RUNX1RUNX1T1, such changes were not consistenly observed. P39 was selected for experimentation, despite its possible contamination with HL-60, an AML cell line[29, 30], because it is the most widely 
employed cell line reminiscent of the biology of MDS used to evaluate responses to hypomethylating agents[31, 32]. With the exception CYFIP2-PLCG2, that was present in the P39 cell line in this study, other fusion genes reported in HL-60 were not observed in the P39 cell line that we acquired[33].

DNA hypermethylation leading to downregulated expression of the tumor suppressor PTEN was observed in cell lines and in primary patient specimens. These changes were predicted to impede the tumor suppressive function of PTEN in the PI3K-AKT-MDM2 signaling pathway[34-36]. In addition, PTEN can function in a phosphatase-independent manner and this includes maintenance of genome stability mediated by mono-ubiquitination of nuclear PTEN and interaction with APC/CDH1 to regulate cell proliferation ${ }^{[36]}$. Reduced expression of PTEN has been reported in high-risk MDS and is related to a constitutive activation of AKT via phosphorylation[36]. A single knockout of PTEN in a murine model was insufficient to produce an MDS phenotype, but PTEN deficiency in combination with SHIP knockout resulted in a severe MDS phenotype. The levels of PTEN and SHIP are the key factors as the two phosphatases share the same substrate $\mathrm{PIP}_{3[37]}$.

In this study, we demonstrated that downregulation of PTEN in HMA resistance induced in vitro was associated with DNA methylation. Interestingly, while PTEN was not methylated in P39, a low level of DNA methylation was sufficient to suppress PTEN transcription in P39-AZA-R and P39-DEC-R. Another mechanism that may be involved in the profound PTEN downregulation observed in addition to DNA methylation might involve the feedback loop of PTEN and p53. A positive feedback mechanism was proposed, with PTEN essential for p53-dependent senescence by maintaining p53 stability, and the PTENp53 tetramer enhancing p53 DNA binding and transcriptional activity[34, 38, 39]

In hematologic malignancies, murine models with PTEN deletions have been observed in association with myeloproliferative neoplasm (MPN), chronic myeloid leukemia (CML) and MDS. However, PTEN downregulation due to genetic or epigenetic alteration has not been previously described in $\mathrm{AML}[36,40]$. In contrast to P39, PTEN remained mostly unmethylated in HMA-sensitive and HMA-resistant Kasumi-1, with only one CpG locus being methylated in Kasumi-DEC-R. In Kasumi-1, PTEN downregulation during AZA / DEC resistance might be related to RUNX1-RUNX1T1 suppression of PTEN via epigenetic silencing of the tumor-suppressive miR-193a[41].

Unlike PTEN that is a positive regulator of p53, the MDM2 protein is a RING finger E3 ubiquitin ligase commonly known to suppress p53[42, 43]. It hetero-dimerizes with MDM4 (also known as MDMX) to enhance p53 mono- or poly-ubiquitination under cellular stress. This facilitates p53 nuclear-tocytoplasmic translocation, with mono-ubiquitination of p53 inhibiting transcriptional activity and polyubiquitination enhanceing p53 degradation in normal cells[42, 43]. In this study, MDM2 was downregulated in all HMA resistant cell lines despite significant DNA hypomethylation. On the other hand, the MDM2 splicing machinery was altered as evidenced by the upregulation of a truncated MDM2 transcript. This suggested that the modulation of MDM2 observed during HMA resistance was dependent on RNA methylation rather than DNA methylation. Since the truncated MDM2 transcript observed does not possess an open reading frame or the RING domain responsible for p53 binding, the biologic 
consequence was downregulation of MDM2 function[44]. Therefore, MDM2 is functionally downregulated with the production of this truncated MDM2 transcript. Multiple studies have reported MDM2 as a bi-functional regulator of p53, and may be involved in a p53-MDM2 feedback loop[45-48]. In situations where MDM2 functions as a TP53 enhancer, MDM2 stimulates TP53 mRNA translation while suppressing its poly-ubiquitination and proteosomal degradation. As expected, MDM2 also promotes degradation of mutated p53 in a similar manner as wild-type p53 in-vivo, and functions as tumorsuppressor to prevent solid tumor formation[45].

MDM2 also possesses multiple function in hematological neoplasms. Firstly, MDM2 is anti-apoptotic, restoring erythroid progenitors from p53-mediated apoptosis. Double knockout of MDM2 in murine models compromised erythropoiesis leading to a phenotype similar to MDS with del(5q), which was lethal during postnatal hematopoiesis[42, 43]. In the context of MDM2 dysregulation, MDM2 mutation at the promoter $\left(M D M 2^{S N P 309}\right)$ has been described in MDS pateints[49]. Co-occurrence of $M D M 2^{S N P 309}$ with the TP53 R72P mutation adversely affected overall survival and progression-free survival in non-del(5q) MDS but not isolated del(5q) MDS[49]. Similar genotypic analyses in AML showed worse outcomes in AML patients with $M D M 2^{S N P 309}$ that was associated with increased MDM2 expression[50]. In contrast, a concomitant downregulation of MDM2 with p53 was observed in MDS with del(5q), with MDM2 responsible for enhancing p53 auto-ubiquitination and degradation[51]. In addition, treatment with lenalidomide induced MDM2 expression that accelerated p53 degradation, suggesting that the function of MDM2 in MDS was complex [51]. Therefore, the role of MDM2 during in myeloid development remains to be better defined.

Given the fact that MDM2 expression was downregulated in all four HMA-resistant sublines in this study and that P39 and Kasumi-1 did not harbor chromosome $5 q$ deletion, both the tumor-suppressive role independent of $5 q$ deletion and the conventional proto-oncogenic role of MDM2 observed in del(5q) MDS should be considered [52]. Because the functional impact of $M D M 2$ on the common myeloid progenitor or granulocyte monocyte progenitor is unknown, we cannot exclude a potential oncogenic role of $M D M 2$ in cell differentiation and within the bone marrow environment[53]. In the context of cell cycle regulation and apoptosis, we hypothesize that the HMA-resistant cells evade apoptosis not only by PTEN suppression but also by MDM2 downregulation, with both PTEN and MDM2 functioning as tumor suppressors. Taking into account the oncogenic role of $M D M 2$ reported in del(5q) MDS and AML, we further propose a functional switch of MDM2 from oncogenic to tumor suppressive during HMA resistance, with loss of MDM2 being attributed to RNA hypomethylation (Fig. 5) [49, 50] In addition, the truncated MDM2 observed in our study only retains the 3' UTR and is in-keeping with a long non-coding RNA without open reading frame[54]. It has been shown that the iron-binding protein IRP2 binds to the 3'UTR of MDM2, which results in stabilization of the MDM2 transcript[55]. We postulate that there is a competitive binding between normal MDM2 and the truncated MDM2 for IRP2, resulting in MDM2 mRNA instability and degradation during HMA resistance. The downregulation of MDM2 was clearly observed on our cell line models despite not too noticeable in patients. This supports our proposition that MDM2 
switched from an oncogene to tumor suppressor gene during treatment with HMA with MDM2 downregulation contributing to HMA resistance.

In conclusion, this study demonstrated that DNA methylation-mediated downregulation of PTEN and epitranscriptional silencing of MDM2 in HMA-resistant cell line are involved in p53 dysregulation via $\mathrm{PI} 3 \mathrm{~K} / \mathrm{Akt} / \mathrm{mTOR}$ signaling. Moreover, this study demonstrated the effect of HMA on RNA methylation contributing to alternative splicing in MDM2. We propose a novel mechanism of p53 dysregulation that involves a functional switch of MDM2 during HMA resistance. Further validation of this model, particularly the role of RNA methylation in HMA-resistance, is warranted. Furthermore, our results also showed oncogenic addiction to the dysregulated PI3K/Akt/mTOR signaling, PTEN and MDM2 during HMA resistance. These may serve as a target for therapeutic modulation by small molecules[56-58].

\section{Availability of data and materials}

All high-throughput sequencing data that supported the findings of this study were deposited in the Gene Expression Omnibus (GEO) with the accession code (GSE165188). For specific dataset, the accession numbers are GSE165185 for methylation microarray data, GSE165187 for RNA transcriptomic data, and GSE165064 for native RNA sequencing data for epi-transcriptomic analysis. Other relevant data supporting the key findings of this study are available within the article or upon request to the corresponding author.

\section{Declarations}

Availability of data and materials

All high-throughput sequencing data that supported the findings of this study were deposited in the Gene Expression Omnibus (GEO) with the accession code (GSE165188). For specific dataset, the accession numbers are GSE165185 for methylation microarray data, GSE165187 for RNA transcriptomic data, and GSE165064 for native RNA sequencing data for epi-transcriptomic analysis. Other relevant data supporting the key findings of this study are available within the article or upon request to the corresponding author.

\section{Acknowledgements}

We would like to acknowledge the Beijing Genomics Institute (BGI) and the Centre for PanorOmic Sciences of the University of Hong Kong Whole methylome analysis, whole transcriptome analysis and pyrosequencing were performed.

Funding support

The work was supported by the General Research Fund (GRF) (project code: 17118914), Research Grant Council (RGC) of Hong Kong Special Administrative Region, China. 
P.L. and R.Y, performed the experiments, wrote the main manuscript text. K-H.M., S-H.F., J.J.L., Z.W., J.L., Y.Y., H-T.C., P-K.Y., and E.Y. performed the experiments. E.T.,Y-L.K wrote and approved the manuscript. H.G. conceived the study and wrote the manuscript. All authors reviewed the manuscript.

Ethics approval and consent to participate

This study was approved by the Institutional Review Board of the University of Hong Kong/ Hong Kong West Cluster (UW 19-634) and written informed consents were obtained.

Consent for publication

Not applicable

Competing interests

The authors have no relevant competing interests to disclose.

\section{References}

1. Walter MJ, Shen D, Ding L, Shao J, Koboldt DC, Chen K, Larson DE, McLellan MD, Dooling D, Abbott R et al: Clonal architecture of secondary acute myeloid leukemia. The New England journal of medicine 2012, 366(12):1090-1098.

2. Woll PS, Kjallquist U, Chowdhury O, Doolittle H, Wedge DC, Thongjuea S, Erlandsson R, Ngara M, Anderson K, Deng Q et al: Myelodysplastic syndromes are propagated by rare and distinct human cancer stem cells in vivo. Cancer Cell 2014, 25(6):794-808.

3. Platzbecker U: Treatment of MDS. Blood 2019, 133(10):1096-1107.

4. Jabbour E, Short NJ, Montalban-Bravo G, Huang X, Bueso-Ramos C, Qiao W, Yang H, Zhao C, Kadia T, Borthakur $\mathrm{G}$ et al: Randomized phase 2 study of low-dose decitabine vs low-dose azacitidine in lower-risk MDS and MDS/MPN. Blood 2017, 130(13):1514-1522.

5. Tobiasson M, Abdulkadir H, Lennartsson A, Katayama S, Marabita F, De Paepe A, Karimi M, Krjutskov $\mathrm{K}$, Einarsdottir E, Grovdal M et al: Comprehensive mapping of the effects of azacitidine on DNA methylation, repressive/permissive histone marks and gene expression in primary cells from patients with MDS and MDS-related disease. Oncotarget 2017, 8(17):28812-28825.

6. Makishima H, Yoshizato T, Yoshida K, Sekeres MA, Radivoyevitch T, Suzuki H, Przychodzen B, Nagata Y, Meggendorfer M, Sanada M et al: Dynamics of clonal evolution in myelodysplastic syndromes. Nat Genet 2017, 49(2):204-212.

7. Itzykson R, Kosmider O, Cluzeau T, Mansat-De Mas V, Dreyfus F, Beyne-Rauzy O, Quesnel B, Vey N, Gelsi-Boyer V, Raynaud S et al: Impact of TET2 mutations on response rate to azacitidine in 
myelodysplastic syndromes and low blast count acute myeloid leukemias. Leukemia 2011, 25(7):1147-1152.

8. Traina F, Visconte V, Elson P, Tabarroki A, Jankowska AM, Hasrouni E, Sugimoto Y, Szpurka H, Makishima H, O'Keefe $\mathrm{CL}$ et al: Impact of molecular mutations on treatment response to DNMT inhibitors in myelodysplasia and related neoplasms. Leukemia 2014, 28(1):78-87.

9. Valencia A, Masala E, Rossi A, Martino A, Sanna A, Buchi F, Canzian F, Cilloni D, Gaidano V, Voso MT et al: Expression of nucleoside-metabolizing enzymes in myelodysplastic syndromes and modulation of response to azacitidine. Leukemia 2014, 28(3):621-628.

10. Qin T, Castoro R, El Ahdab S, Jelinek J, Wang X, Si J, Shu J, He R, Zhang N, Chung W et al: Mechanisms of resistance to decitabine in the myelodysplastic syndrome. PLoS One 2011, 6(8):e23372.

11. Santini V: How I treat MDS after hypomethylating agent failure. Blood 2019, 133(6):521-529.

12. Meldi K, Qin T, Buchi F, Droin N, Sotzen J, Micol JB, Selimoglu-Buet D, Masala E, Allione B, Gioia D et al: Specific molecular signatures predict decitabine response in chronic myelomonocytic leukemia. $J$ Clin Invest 2015, 125(5):1857-1872.

13. The Gene Ontology C: The Gene Ontology Resource: $\mathbf{2 0}$ years and still GOing strong. Nucleic Acids Res 2019, 47(D1):D330-D338.

14. Kanehisa M, Goto S: KEGG: kyoto encyclopedia of genes and genomes. Nucleic Acids Res 2000, 28(1):27-30.

15. Chen Y, Chen Y, Shi C, Huang Z, Zhang Y, Li S, Li Y, Ye J, Yu C, Li Z et al: SOAPnuke: a MapReduce acceleration-supported software for integrated quality control and preprocessing of high-throughput sequencing data. Gigascience 2018, 7(1):1-6.

16. Kim D, Paggi JM, Park C, Bennett C, Salzberg SL: Graph-based genome alignment and genotyping with HISAT2 and HISAT-genotype. Nat Biotechnol 2019, 37(8):907-915.

17. Pertea M, Pertea GM, Antonescu CM, Chang TC, Mendell JT, Salzberg SL: StringTie enables improved reconstruction of a transcriptome from RNA-seq reads. Nat Biotechnol 2015, 33(3):290-295.

18. Trapnell C, Williams BA, Pertea G, Mortazavi A, Kwan G, Van Baren MJ, Salzberg SL, Wold BJ, Pachter L: Transcript assembly and quantification by RNA-Seq reveals unannotated transcripts and isoform switching during cell differentiation. Nature Biotechnology 2010, 28(5):511-515.

19. Langmead B, Salzberg SL: Fast gapped-read alignment with Bowtie 2. Nat Methods 2012, 9(4):357359.

20. Li B, Dewey CN: RSEM: accurate transcript quantification from RNA-Seq data with or without a reference genome. BMC Bioinformatics 2011, 12:323.

21. Team RC: R: A Language and Environment for Statistical Computing. In. Vienna, Austria: R Foundation for Statistical Computing; 2013.

22. Ashburner M, Ball CA, Blake JA, Botstein D, Butler H, Cherry JM, Davis AP, Dolinski K, Dwight SS, Eppig JT et al: Gene ontology: tool for the unification of biology. The Gene Ontology Consortium. Nat 
Genet 2000, 25(1):25-29.

23. Mi H, Muruganujan A, Ebert D, Huang X, Thomas PD: PANTHER version 14: more genomes, a new PANTHER GO-slim and improvements in enrichment analysis tools. Nucleic Acids Res 2019, 47(D1):D419-D426.

24. Jia W, Qiu K, He M, Song P, Zhou Q, Zhou F, Yu Y, Zhu D, Nickerson ML, Wan S et al: SOAPfuse: an algorithm for identifying fusion transcripts from paired-end RNA-Seq data. Genome Biol 2013, 14(2):R12.

25. Krzywinski M, Schein J, Birol I, Connors J, Gascoyne R, Horsman D, Jones SJ, Marra MA: Circos: an information aesthetic for comparative genomics. Genome Res 2009, 19(9):1639-1645.

26. Li H: Minimap2: pairwise alignment for nucleotide sequences. Bioinformatics 2018, 34(18):30943100 .

27. Loman NJ, Quick J, Simpson JT: A complete bacterial genome assembled de novo using only nanopore sequencing data. Nature Methods 2015, 12(8):733-U751.

28. Pratanwanich PN, Yao F, Chen Y, Koh CWQ, Hendra C, Poon P, Goh YT, Yap PML, Yuan CJ, Chng WJ et al: Detection of differential RNA modifications from direct RNA sequencing of human cell lines. In.: Cold Spring Harbor Laboratory; 2020.

29. Steensma DP: P39/Tsugane cells are a false cell line contaminated with HL-60 cells and are not suitable for mechanistic studies in myelodysplastic syndromes. Haematologica 2010, 95(7):12291230.

30. Drexler HG, Dirks WG, Macleod RA: Many are called MDS cell lines: one is chosen. Leuk Res 2009, 33(8):1011-1016.

31. Khan R, Aggerholm A, Hokland P, Hassan M, Hellstrom-Lindberg E: A pharmacodynamic study of 5azacytidine in the P39 cell line. Exp Hematol 2006, 34(1):35-43.

32. Tsujioka T, Yokoi A, Uesugi M, Kishimoto M, Tochigi A, Suemori S, Tohyama Y, Tohyama K: Effects of DNA methyltransferase inhibitors (DNMTIs) on MDS-derived cell lines. Exp Hematol 2013, 41(2):189-197.

33. Jacobson EC, Grand RS, Perry JK, Vickers MH, Olins AL, Olins DE, O'Sullivan JM: Hi-C detects novel structural variants in HL-60 and HL-60/S4 cell lines. Genomics 2020, 112(1):151-162.

34. Chen Z, Trotman LC, Shaffer D, Lin HK, Dotan ZA, Niki M, Koutcher JA, Scher HI, Ludwig T, Gerald W et al: Crucial role of p53-dependent cellular senescence in suppression of Pten-deficient tumorigenesis. Nature 2005, 436(7051):725-730.

35. Zheng T, Meng X, Wang J, Chen X, Yin D, Liang Y, Song X, Pan S, Jiang H, Liu L: PTEN- and p53mediated apoptosis and cell cycle arrest by FTY720 in gastric cancer cells and nude mice. J Cell Biochem 2010, 111(1):218-228.

36. Morotti A, Panuzzo C, Crivellaro S, Carra G, Torti D, Guerrasio A, Saglio G: The Role of PTEN in Myeloid Malignancies. Hematol Rep 2015, 7(4):5844. 
37. Moody JL, Xu L, Helgason CD, Jirik FR: Anemia, thrombocytopenia, leukocytosis, extramedullary hematopoiesis, and impaired progenitor function in Pten+/-SHIP-/- mice: a novel model of myelodysplasia. Blood 2004, 103(12):4503-4510.

38. Kim J, Eltoum IEA, Roh M, Wang J, Abdulkadir SA: Interactions between Cells with Distinct Mutations in C-MYC and Pten in Prostate Cancer. Plos Genet 2009, 5(7).

39. Li AG, Piluso LG, Cai X, Wei G, Sellers WR, Liu X: Mechanistic insights into maintenance of high p53 acetylation by PTEN. Mol Cell 2006, 23(4):575-587.

40. Cancer Genome Atlas Research N, Ley TJ, Miller C, Ding L, Raphael BJ, Mungall AJ, Robertson A, Hoadley K, Triche TJ, Jr., Laird PW et al: Genomic and epigenomic landscapes of adult de novo acute myeloid leukemia. N Engl J Med 2013, 368(22):2059-2074.

41. Li Y, Gao L, Luo X, Wang L, Gao X, Wang W, Sun J, Dou L, Li J, Xu C et al: Epigenetic silencing of microRNA-193a contributes to leukemogenesis in $t(8 ; 21)$ acute myeloid leukemia by activating the PTEN/PI3K signal pathway. Blood 2013, 121(3):499-509.

42. Liu G, Terzian T, Xiong S, Van Pelt CS, Audiffred A, Box NF, Lozano G: The p53-Mdm2 network in progenitor cell expansion during mouse postnatal development. J Pathol 2007, 213(4):360-368.

43. Cumbo C, Tota G, Anelli L, Zagaria A, Specchia G, Albano F: TP53 in Myelodysplastic Syndromes: Recent Biological and Clinical Findings. Int J Mol Sci 2020, 21(10).

44. Hunt SE, McLaren W, Gil L, Thormann A, Schuilenburg H, Sheppard D, Parton A, Armean IM, Trevanion SJ, Flicek P et al: Ensembl variation resources. Database (Oxford) 2018, 2018.

45. Manfredi JJ: The Mdm2-p53 relationship evolves: Mdm2 swings both ways as an oncogene and a tumor suppressor. Gene Dev 2010, 24(15):1580-1589.

46. Hernandez-Monge J, Rousset-Roman AB, Medina-Medina I, Olivares-Illana V: Dual function of MDM2 and MDMX toward the tumor suppressors p53 and RB. Genes Cancer 2016, 7(9-10):278-287.

47. Hu B, Gilkes DM, Chen J: Efficient p53 activation and apoptosis by simultaneous disruption of binding to MDM2 and MDMX. Cancer Res 2007, 67(18):8810-8817.

48. Ye J, Liang R, Bai T, Lin Y, Mai R, Wei M, Ye X, Li L, Wu F: RBM38 plays a tumor-suppressor role via stabilizing the p53-mdm2 loop function in hepatocellular carcinoma. J Exp Clin Cancer Res 2018, 37(1):212.

49. McGraw KL, Cluzeau T, Sallman DA, Basiorka AA, Irvine BA, Zhang L, Epling-Burnette PK, Rollison DE, Mallo M, Sokol L et al: TP53 and MDM2 single nucleotide polymorphisms influence survival in nondel(5q) myelodysplastic syndromes. Oncotarget 2015, 6(33):34437-34445.

50. Falk IJ, Willander K, Chaireti R, Lund J, Nahi H, Hermanson M, Green H, Lotfi K, Soderkvist P: TP53 mutations and MDM2(SNP309) identify subgroups of AML patients with impaired outcome. Eur J Haematol 2015, 94(4):355-362.

51. Wei S, Chen X, McGraw K, Zhang L, Komrokji R, Clark J, Caceres G, Billingsley D, Sokol L, Lancet J et al: Lenalidomide promotes p53 degradation by inhibiting MDM2 auto-ubiquitination in myelodysplastic syndrome with chromosome 5q deletion. Oncogene 2013, 32(9):1110-1120. 
52. Ogawa S: Genetics of MDS. Blood 2019, 133(10):1049-1059.

53. Pleyer L, Valent P, Greil R: Mesenchymal Stem and Progenitor Cells in Normal and Dysplastic Hematopoiesis-Masters of Survival and Clonality? Int J Mol Sci 2016, 17(7).

54. Mercer TR, Dinger ME, Mattick JS: Long non-coding RNAs: insights into functions. Nat Rev Genet 2009, 10(3):155-159.

55. Zhang J, Kong X, Zhang Y, Sun W, Xu E, Chen X: Mdm2 is a target and mediator of IRP2 in cell growth control. FASEB J 2020, 34(2):2301-2311.

56. McCubrey JA, Steelman LS, Abrams SL, Bertrand FE, Ludwig DE, Basecke J, Libra M, Stivala F, Milella $M$, Tafuri $A$ et al: Targeting survival cascades induced by activation of Ras/Raf/MEK/ERK, PI3K/PTEN/Akt/mTOR and Jak/STAT pathways for effective leukemia therapy. Leukemia 2008, 22(4):708-722.

57. Steelman LS, Abrams SL, Whelan J, Bertrand FE, Ludwig DE, Basecke J, Libra M, Stivala F, Milella M, Tafuri A et al: Contributions of the Raf/MEK/ERK, PI3K/PTEN/Akt/mTOR and Jak/STAT pathways to leukemia. Leukemia 2008, 22(4):686-707.

58. Morris LG, Chan TA: Therapeutic targeting of tumor suppressor genes. Cancer 2015, 121(9):13571368.

\section{Figures}

\section{Figure 1}

A

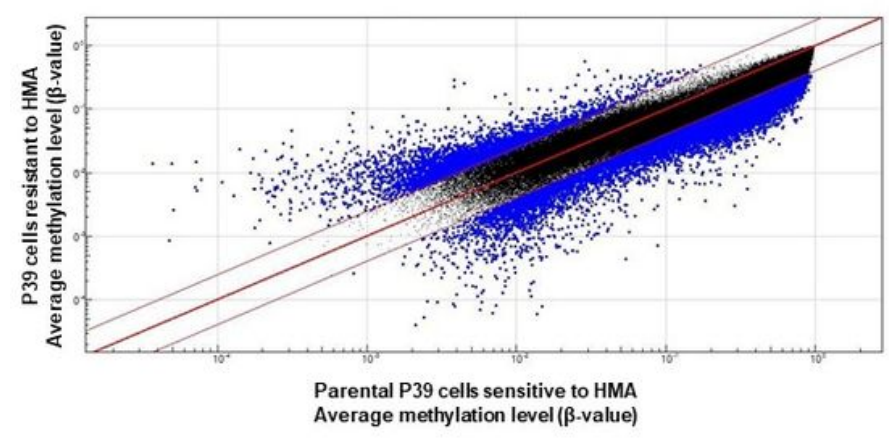

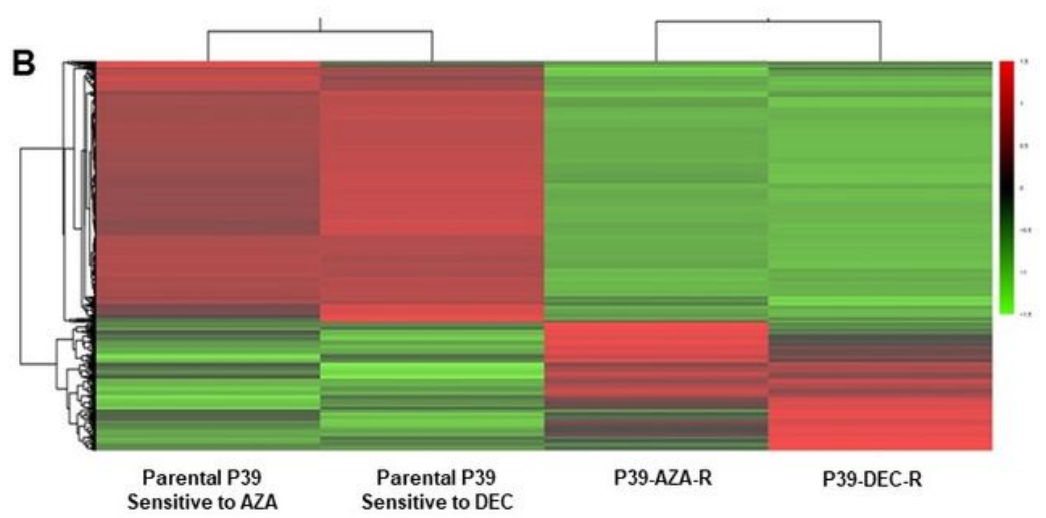

Figure 1 
Differential methylation signature between hypomethylating agent (HMA)-resistant and sensitive P39

cells. A: Scatter plot of differential methylation loci of hypomethylating agent-resistant and sensitive P39 cell lines. Average methylation levels of P39 cell line resistant to azacitidine and decitabine were compared with the mean methylation levels of P39 cells sensitive to azaditidine and decitabine. Differential methylation levels of at least 2.5 -fold or 0.4 -fold were included for downstream study as differentially methylated regions (DMRs); B: Differential methylation signature and functional prediction of differentially methylated regions (DMRs) in HMA-sensitive and HMA-resistant cells. Hierarchical clustering of DMRs showed a global hypomethylation signature during resistance to both azacitidine (AZA) and decitabine (DEC). Methylation in AZA-resistant P39 cell line (P39-AZA-R) and DEC-resistant P39 cell line (P39-DEC-R) differed by a small number of hypermethylated genes. The sensitive parental P39 cells were treated separately with AZA and DEC at $1 \mu \mathrm{M}$ for 48 hours followed by immediate harvest for the assessment of methylation signature.

\section{Figure 2}

A

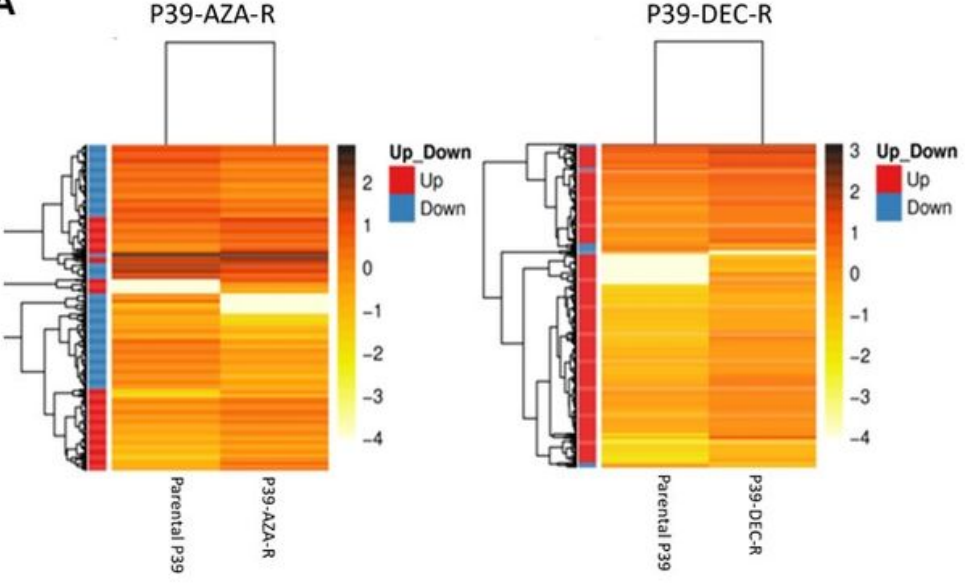

B
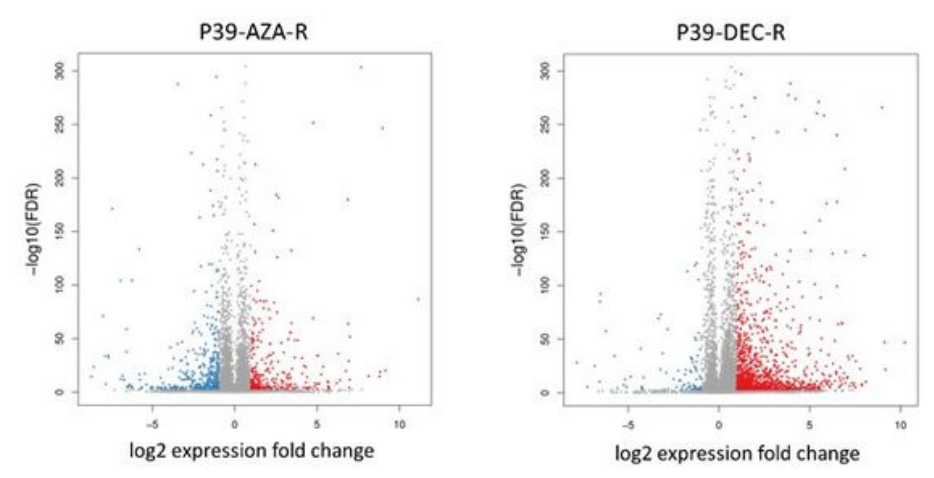

Figure 2

Differential expression profile of hypomethylating agent (HMA)-resistant P39 cells. A: RNA expression profiles of AZA-resistant P39 (P39-AZA-R) and DEC-resistant P39 (P39-DEC-R) were distinct from each other with different clustering pattern; B: Volcano plot of differentially expressed genes. Overexpression of genes was seen in P39-DEC-R and P39-AZA-R when compared with P39. The upregulated genes (blue) correspond to their negative log10 false discovery ratio (FDR) (p-value). P39-DEC-R (right) detected 1296 significantly upregulated genes (red dots) while P39-AZA-R (left) detected 306 significantly upregulated 
genes when compared with P39. There were 420 downregulated genes detected in P39-AZA-R and 89 downregulated genes detected in P39-DEC-R in comparison with P39.

\section{Figure 3}
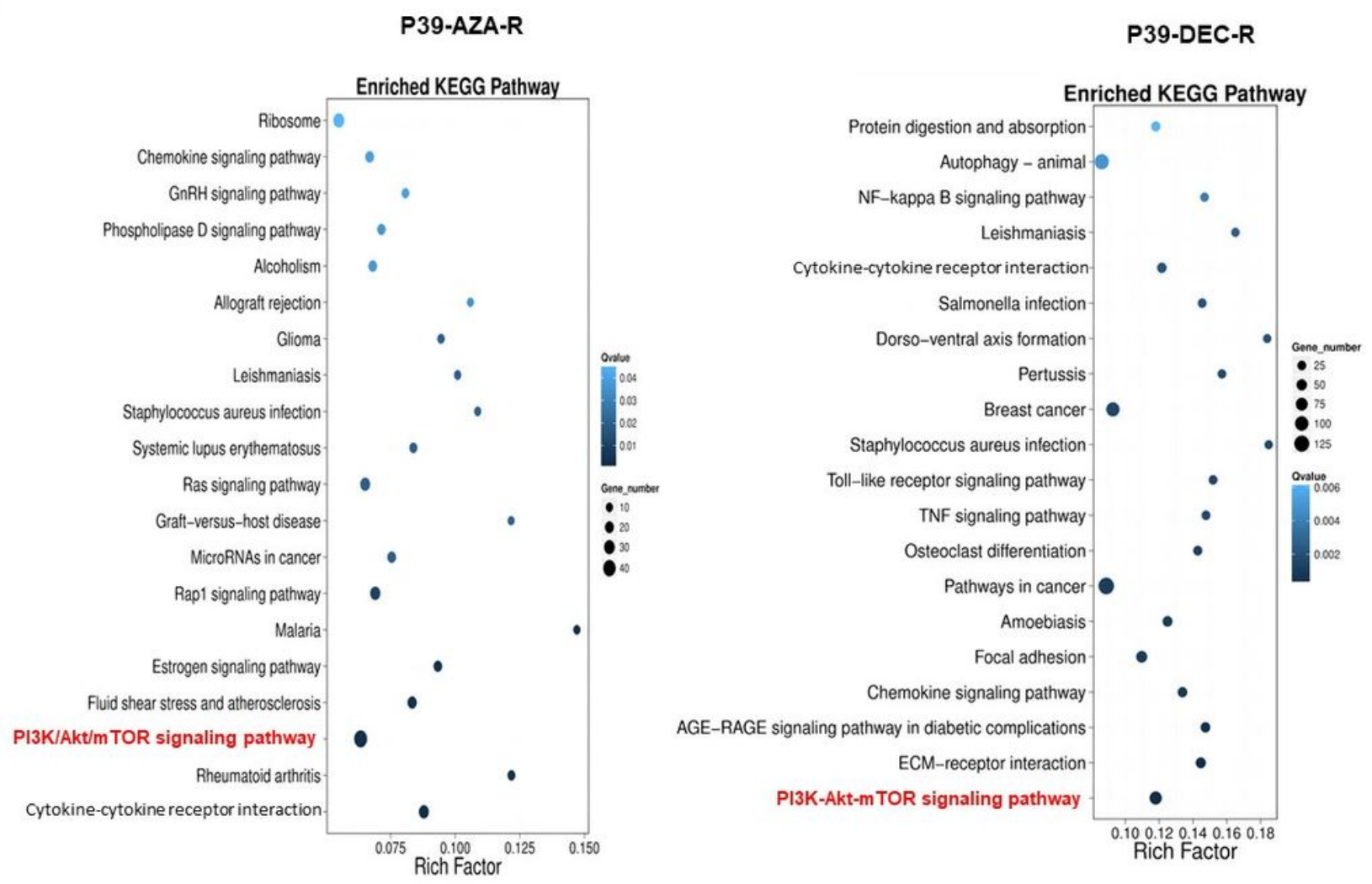

\section{Figure 3}

\section{KEGG prediction for pathway and functional roles of differentially expressed genes (DEGs). Functional} prediction by KEGG analysis of differentially expressed genes in hypomethylating agent (HMA)-resistant P39 sublines compared with P39 was performed separately for azacitidine-resistant P39 (P39-AZA-R) and decitabine-resistant P39 (P39-DEC-R). This gene set analysis studied both over-expressed and underexpressed genes together to look for biological pathways that are implicated. Both HMA-resistant sublines had dysregulated PI3K/Akt signaling that were predicted to be mediated by Platelet Derived Growth Factor Subunit A (PDGFA) and Endothelia PAS domain-containing protein 1 (EPAS1) upon correlation with DNA methylation patterns. Both sublines also showed similar dysregulation in cellular processes. The PI3K-Akt signaling pathway (red) was functionally significant in both cell lines. The result from this RNA expression-based analysis support the $850 \mathrm{~K}$ methylome data that predicted PI3K (PTEN and MDM2) signaling dysregulation during HMA resistance.

Rich factor: ratio of the DEG number and the number of genes annotated in the relevant pathway. The greater of the Rich factor, the greater the degree of significance of the pathway identified.

KEGG: Kyoto Encyclopedia of Genes and Genomes. 
Figure 4

A
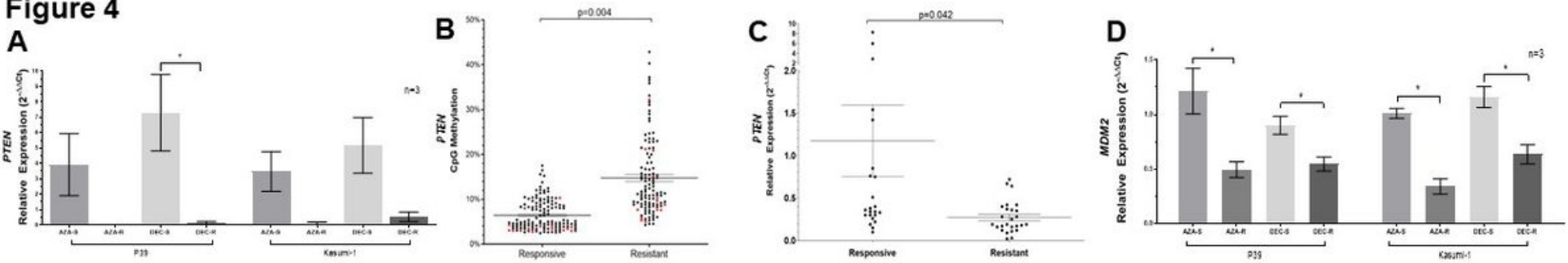

E

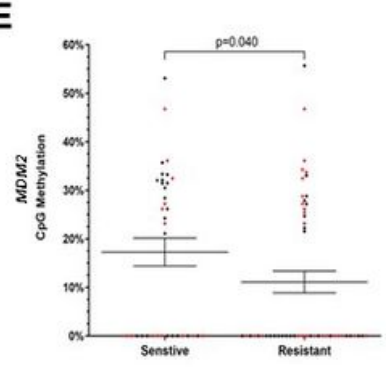

$\mathbf{F}$

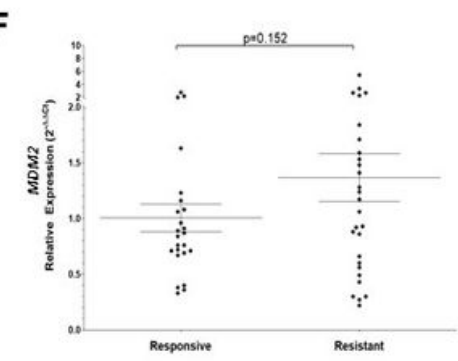

G
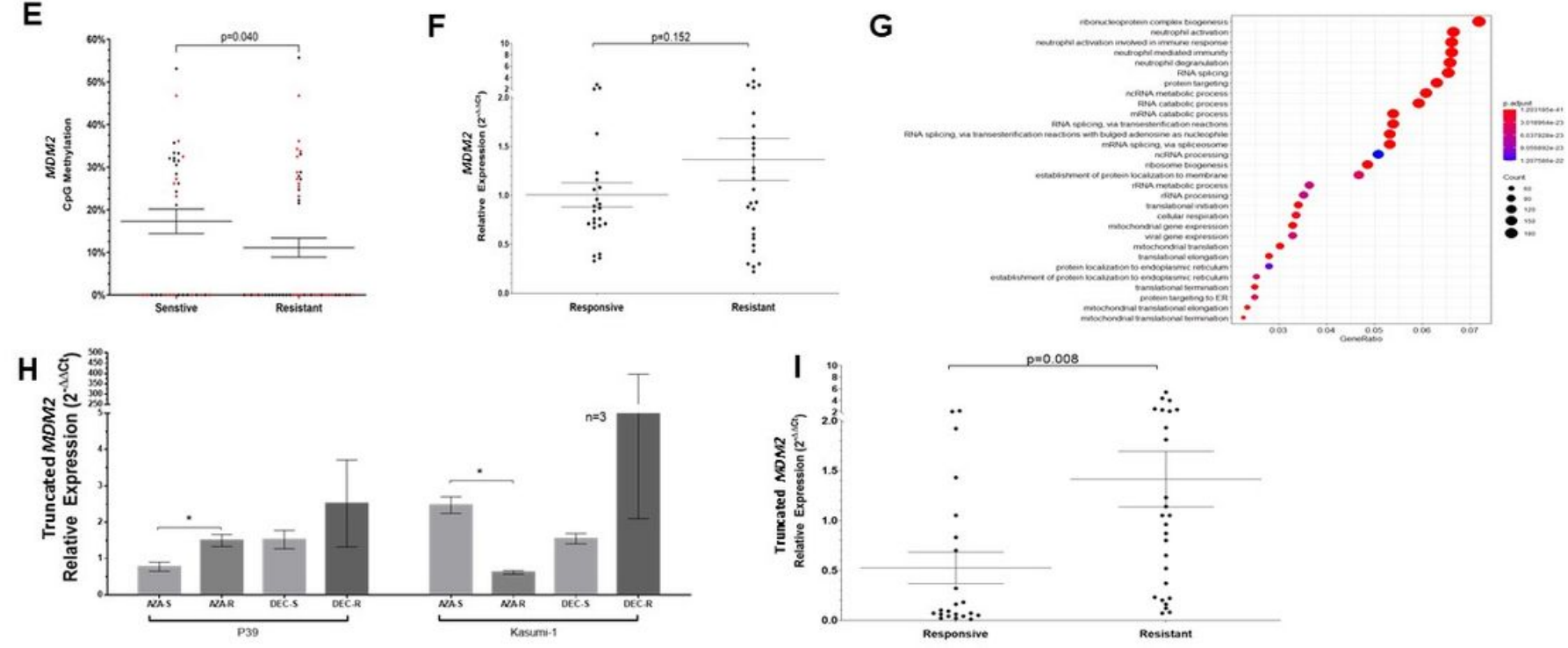

Figure 4

Association of PTEN and MDM2 methylation with mRNA expression. A: Downregulation of PTEN by quantitative polymerase chain reaction (Q-PCR) in azacitidine and decitabine resistant cell lines; $\mathbf{B}$ : Validation of PTENDNA methylation in patients responseive or resistant to hypomethylating agents using pyrosequencing. Individual CpG methylation levels were displayed as individual datapoints with red data points (red dots) being the same loci (cg10041390) detected to be hypermethylated in 850k whole methylome microarray. The total number of dots denotes the number of CpG sites evaluated, where red dots denote $\mathrm{CpG}$ sites with methylation changes and black dots denote flanking or additional CpG sites; C: Downregulation of PTEN by Q-PCR in patients with myelodysplastic resistant to hypomethylating agents; D: Expression of MDM2 in cell lines sensitive and resistant to azacitidine and decitabine; E: Validation of MDM2 hypomethylation in patients with MDS resistant to hypomethylating agents by pyrosequencing. Individual CpG methylation levels were displayed as individual datapoints with red data points (red dots) being the same loci (cg00614420) detected to be hypomethylated in 850k whole methylome microarray. The total number of dots denotes the number of CpG sites evaluated where red dots denote $\mathrm{CpG}$ sites with methylation changes and black dots denote flanking or additional CpG sites ; F: Expression of MDM2 in patients with MDS responsive and resistant to hypomethylating agents; G: Epitranscriptomic profiling of P39 and azacitidine-resistant P39 (P39-AZA-R) cell line. Direct RNA sequencing of P39 cells and P39-AZA-R detected 5,340,619 differential methylated transcripts including the MDM2 processed transcript. Gene Ontogeny (GO) analysis demonstrated that the most enriched GO term comprised transcripts involved in ribonucleoprotein biogenesis and neutrophil functions; $\mathrm{H}$ : Increased expression of the MDM2 truncated transcript was detected in P39-AZA-R but was downregulated in 
Kasumi-AZA-R compared with corresponding parental cells ( $p<0.05$ for both); I: Increased expression of the MDM2 processed transcript in patients with myelodysplastic syndrome resistant to hypomethylating agents. Horizontal lines: mean \pm standard error of mean (SEM); Unpaired t-tests were performed for statistical analysis and two-tailed $p$-values $<0.05$ were considered statistically significant; *: $p<0.05$. P39 cells were treated separately with AZA and DEC at $1 \mu \mathrm{M}$ for 48 hours followed by immediate harvest for the assessment of methylation status and mRNA expression.

Figure 5
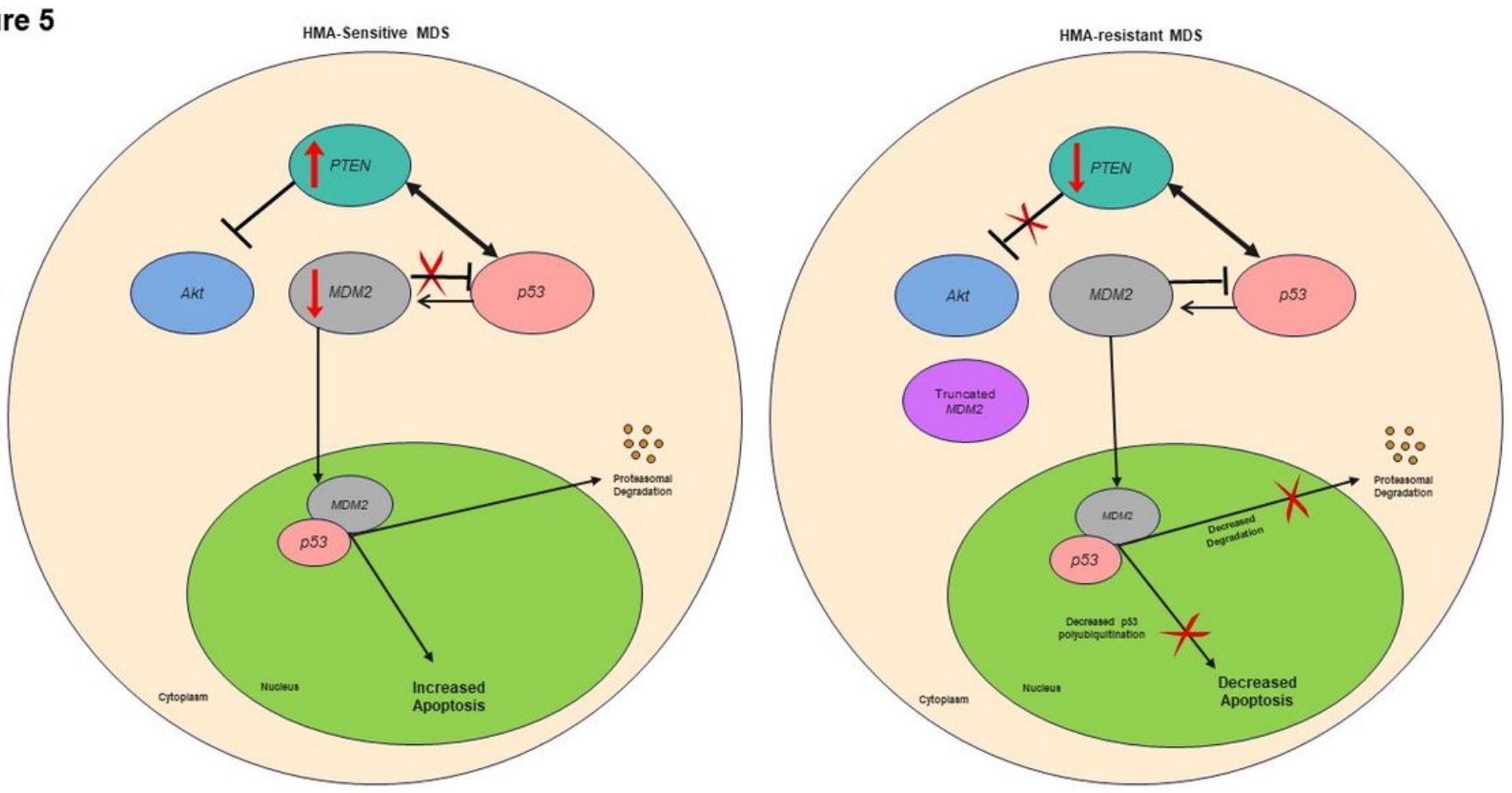

\section{Figure 5}

Proposed molecular mechanism of resistance to hypomethylating agents involving PTENand MDM2. During resistance to hypomethylating agents, further suppression of PTEN is associated with CPG DNA hypermethylation. RNA M6A hypomethylation led to unexpected alternative splicing, which resulted in up-regulation of truncated MDM2 mRNA as non-coding transcript with only the 3 ' untranslated region. PTEN functions as a tumor suppressor gene and inhibits PI3K/Akt signaling. This inhibition is lost upon DNA hypermethylation of PTEN during HMA-resistance. On the other hand, a shift in the function of MDM2 could be associated with HMA-resistance, with MDM2 initially being an oncoprotein conventionally known to negatively regulate p53. During HMA resistance, MDM2 is switched into a p53 enhancer but the expression of $M D M 2$ was suppressed due to RNA hypomethylation and altered MDM2 splicing. Therefore, PTEN and MDM2 silencing collectively result in dysregulated p53.

\section{Supplementary Files}


This is a list of supplementary files associated with this preprint. Click to download.

- Supplementalfileslegends.docx

- Supplementalfile1.xlsx

- Supplementalfile2.pptx

- Supplementalfile3.pptx

- Supplementalfile4.docx

- Supplementalfile5.xlsx

- Supplementalfile6.xlsx

- Supplementalfile7.pptx

- Supplementalfile8.xlsx

- Supplementalfile9.xlsx

- Supplementalfile10.pptx

- Supplementalfile11.pptx

- Supplementalfile12.pptx

- Supplementalfile13.pptx

- Supplementalfile14.pptx

- Supplementalfile15.pptx

- Supplementalfile16.xlsx 